ste iner-Wien, Op pen heim-Berlin, Oppenheim-Frankfurta/M., Pappenheim-Wien, Peters-Conradstein, Peipers-Bonn, Peltzer-Bremen, PeritzBerlin, Phleps-Graz, Pick-Prag, Poensgen-Bochum, Powers-S. Franzisco, Quensel-Leipzig, Raut enberg-Hamburg, R eckmann-Oeynhausen, Redlich-Wien, Rehm-Blankenburg, Reinhold-Hannover, ResnikowCharkow, Reye-Hamburg, Rittershaus-Hamburg, Römer-Hirsau, Römheld-Hornegg, Rothmann-Berlin, Rullah-Wien, Rumpf-Eonn, SachsHamburg, Saenger-Hamburg, Schilder-Leipzig, Schlesinger-Wien, Sehlesiner-Berlin, FurtSchmidt-Dresden, Schönborn-Heidelberg, Schüller-Wien, Schuster-Aachen, Schuster-Berlin, SchultzeKahleyss-Hofheim, Schwabe-Plauen, Schwarz-Riga, Schwenckenbe cher-Frankfurt a/M., Seeligmann-Hamburg, Seeligmüller-Halle, Simons-Berlin, Sinn-Neubabelsberg, Ste iner-Strabburg, Stender-Riga, Ster $n$-Berlin, S tew art-London, S teyert ha - Kleinen, Teuscher-Weisser Hirsch, Thom sen-Bonn, T imme-New-York, Trömner-Hamburg, To bi asBerlin, Ve it-Wuhlgarten, v. Velzen-Joachimsthal, Vogt-Wiesbaden, Volland-Bethel, Wah rend orff-Ilten, Wallen berg-Danzig, Welm-Ulbrichshöhe, W em er - Hamburg, We st phal-Bonn, Wey g a nd t-Hamburg, W is weSt. Blasien, Wohlwill-Hamburg, Zen di gr-Hamburg.

Als Schriftführer fungiert Herr K. Mendel (Berlin), als lokale Schriftführer die Herren Lüttge und Holzmann (Hamburg).

\title{
Erster Tag.
}

\section{Sitzung.}

Freitag, 27. September, vormittags 9 Uhr.

Vorsitzender: Herr H. Oppenheim.

Der I. Vorsitzende der Gesellschaft, Herr H. Oppenheim, eröffnet die Versammlung mit folgender Ansprache:

\section{Verehrte Herren Kollegen!}

Ich eröffne hiermit die sechste Jabresversammlung der G. D. N. und heisse Sie alle herzlich willkommen. Ein reiches Programm liegt vor uns, in dem die verschiedensten Forschungsrichtungen unserer Spezialwissenschaft vertreten sind. Besonders dürfen wir es begrüssen, dass die Referate von so berufenen Forschern übernommen sind, und ich bitte um die Erlaubnis, schon beim Eintritt in unsere Tagung den Herrn Referenten den Dank unserer Gesellschaft dafür abzustatten, dass sie unserer Aufforderung, hier über die klinische Stellung der genuinen Epilepsie und den gegenwärtigen Stand der Lehre vom Sympathicus alles Wissenswerte und das von ihnen selbst Erforschte mitzuteilen, in so bereitwilliger Weise gefolgt sind.

Die Teilnahme an unseren Jahrestersammlungen wird wohl in 
erster Linie durch den Inhalt des wissenschaftlichen Programms bestimmt. Aber daneben übt der Ort, an dem wir zusammentreffen, eine durch seine Bedentung und Lage bedingte mehr oder weniger beträchtliche Anziehung aus. Dass die Wahl von Hamburg in dieser Hinsicht eine glückliche gewesen ist, dass es für uns alle etwas Verlockendes gehabt hat, hierher zu kommen, das bedarf keiner Begründung, dafür zeugt ja auch die grosse Zahl der Teilnehmer an unserer diesmaligen Jahresversammlung.

Es ist nicht allein die Grösse, der Reichtum, die Schönheit, welche Hamburg eine so hervorragende Stellung unter den Städten des Reiches, unter den Städten Europas verleiht - es ist der besondere Geist, der in dieser: alten Hansastadt steckt, das Freie, Starke, Grosse, Energische Kühne, 'Tüchtige, das in dem Geiste seiner Bürger, in allen Einrichtungen und Unternehmungen zur Geltung kommt. Nach der Gesinnung und dem $W$ esen der Einwohner, nach seiner Geschichte und politischen Stellung eine echt deutsche Stadt, aber durch die Strassen des Meeres, durch seine mächtige Handelsflotte mit dem ganzen Erdball verknüpft, in stetem, lebendigem Anstausch mit allen Bezirken der Welt -: ein echtes Wahrzeichen der freien Wissenschaft, die zwar von dem Geiste eines jeden Volkes beeinflusst wird, von ihm ein bestimmtes Gepräge erhält, aber doch über alle natürlichen und politischen Grenzen hinausgreift, alle Länder und Völker verknüpit, alle Nationen zu dem grossen Volke der nach Erkenntnis und Wahrheit strebenden Menschheit zusammenfasst.

Und wenn die Bücher, die wissenschaftlichen Zeitschriften und Archive in diesem geistigen Verkehr die Schiffe bilden, die mit Schätzen beladen hinüber- und herübersegeln, so darf das mediziniseh-wissenschaftliche Hamburg sich ruihmen, in diesem Weltverkehr eine grosse, angesehene Empore zu bilden.

Ganz besonders gilt das für unser Spezialfach, für die Neurologie, die hier seit Jahrzehnten eine nachahmenswerte Pflege und Förderung erfahren hat. Teh brauche nur den Narnen Eisenlohr zu nennen, um Sie an einen der besten Vertreter unseres Faches zu erinnern, dem die Nerrenheilkunde eine Fülle von Beobachtungen und Resultaten verdankt.

Von den lebenden. Vertretern unseres Faches branche ich nicht zu sprechen, es ist Ihnen allen bekannt, welch bedeutenden Anteil die Hamburger Neurologen an dem Ausbau, an der Fortentwicklung der Nervenheilkunde haben; auch unsere Jahresversammlung wird dafür in beredter Weise Zeugnis ablegen.

M. H.! Wie treten unter günstigen Auspizien in unsere sechste Jahresversammlung. Freilich ist das Ziel, das von unserer Gesell- 
schaft erstrebt wird: die Vertretung der Nervenheilkunde an den grösseren Universitäten und Krankenhäusern durch Nervenärzte, noch unerreicht, und gerade das verflossene Jahr hat uns wieder vor Augen geführt, wie wenig hier zu erwarten ist, solange es uns an der Macht fehlt, die durch die persönlichen Beziehungen bedingt wird.

Aber wenn unsere Gesellschaft weiter wie bisher nach innen erstarkt, wenn sie ihre Prinzipien hochhält und verteidigt, so kann es nicht fehlen, dass der dumpfe Widerstand allmählich besiegt und der Neurologie die Stellung eingeräumt wird, die die Otologie, Laryngologie und Pädiatrie seit langem besitzen.

M. H.! Unsere Gesellschaft hat vor wenigen Wochen einen schmerzlichen Verlust durch den Tod von August Cramer erlitten. Es war nicht nur eine äussere, durch die Mitgliedschaft bedingte Zusammengehörigkeit, nein $\mathrm{Cramer}$ war auch dadurch mit uns verbunden, dass er nach seinem Forschen und Wirken, man darf wohl sagen: mit der dimidia pars animae der Neurologie zugetan war. Sein Werk über die Neurasthenie, seine erst vor kuxzem erschienene Darstellung der Neurosen im Lehrbuch der Nervenkrankheiten des Kindesalters und nicht zum wenigsten die von ihm angeregte Gründung der Nervenheilstätte Rasemühle bei Göttingen zeugen dafür, dass er sich mit Liebe und mit tatkräftigem Erfolg der Nervenheilkunde gewidmet hat. Sein Hauptinteresse und der rorwiegende Teil seiner Lehrtätigkeit galt jedoch der Psychiatrie und hier fesselte ihn wohl wieder am meisten die forensische Seite und das Grenzgebiet der psychopathischen Zustände. In dem bekannten Werke der „Gerichtlichen Psychiatrie für Mediziner und Juristen" hat ex einen grossen Teil seiner Erfahrungen und Anschauungen niedergelegt. Seinem warmen Interesse für die Geisteskranken und der sozialen Fürsorge Bedürftigen verdankt die Provinz Hannover eine Reihe von Institutionen. Die Verehrung und Anerkennung, die er sich an diesen Stätten seines Wirkens erworben bat, ist gelegentlich seiner Berufung nach Berlin and dann wieder bei seinem Ableben zum Ausdruck gekommen.

Im Namen unserer Gesellschaft hat Kollege Bruns einen Kranz an seiner Bahre niedergelegt. Lassen Sie uns heute unsere Anteilnahme an der Traver noch einmal bekunden: Tch bitte Sie sich im. Andenken an den Verstorbenen von den Sitzen zu erheben (geschieht).

lch habe noch eines Mannes zu gedenken, der zwar nicht unserer Gesellschaft angehörte, dessen Tod uns aber doch tief berührt, da er einer der Fürsten unserer Wissenschaft, einer unserer Lehrer and Fiihrer war. Am 7. Oktober vorigen Jahres verschied Hughlings Jackson im Alter ron 76 Jahren, nachdem er ungefähr ein halbes Jahrhundert der Neurologie gewidmet und sich ihr als Forscher, 
Lehrer und Arzt in überaus fruchtbringender Weise gewidmet hatte. Unsere englischen Kollegen nannten ihn the neurologists neurologist, den Nervenarzt aller Nervenärzte, und sagten von ihm, dass die englische Neurologie und Jackson an einem Tage geboren seien. Ihnen allen bekannt ist er besonders durch die nach ihm benannten rindenepileptischen Krämpfe und durch den Nachweis ihrer Beziehungen zu bestimmten Zentren der Hirnrinde. Hitzig selbst sagt von dieser Entdeckung in der Second Hughlings Jackson Lecture (Berlin 1901), „dass er (Jackson) zuerst es war, der die partiellen kortikalen. Krämpfe auf eigene Innervationszentren bezog und auf diese Weise auf die Existenz von motorischen Zentren im Grosshirn schloss, ja sogar ihre ungefähre Lage angab, schon bevor die physiologische Forschung diesen Nachweis mit Sicherheit zu führen vermochte."

Damit hat er sich ein unvergängliches Denkmal gesetzt. Auf die zahlreichen weiteren Beiträge zur Physiologie und Pathologie des Gehirns will ich nicht eingehen.

Dieser ausgezeichnete Forscher war zugleich ein von seinen Schïlern hochverehrter und bewunderter Lehrer, ein von seinen Kranken geschätzter und geliebter Nervenarzt. So haben wir berechtigten Anlass, an der Trauer der englischen Kollegen um das Hinscheiden eines unserer Führer Anteil zu nehmen. lch bitte Sie, sich zu seinem Andenken von den Sitzen zu erheben.

Nun zu den Lebenden!

Die Zahl unserer ordentlichen Mitglieder beträgt z. Z. 419, die der Ehren- und korresp. Mitglieder 29.

Die finanzielle Lage ist trotz grösserer Zuwendungen für wissenschaftliche Zwecke eine günstige.

Das Amt als lokale Sehriftführer haben die Herren Holzmann und Lüttge übernommen.

Mit dem Wunsche, dass unsere Jahresversammlung von dem Geiste der früheren beseelt sei, eröffne ich nunmehr die Sitzung.

Exzellenz Erb hat telegraphisch sein Bedauern ansgedrückt, durch Krankheit verhindert zu sein, an der diesjährigen Tagung teilzunehmen. Mit Zustimmung der Anwesenden wird ihm folgendes Telegramm gesandt:

„Die Gesellschaft Deutscher Nervenärzte vermisst Sie schmerzlich auf ihrer Jahresversammlnng und wünscht lhnen von Herzen gute Besserung."

Herr Stransky (Wien) hat telegraphisch sein Bedauern ausgedrückt, nicht nach Hamburg kommen zu können. 
$2 \Downarrow 6$ Sechste Jahresversammlung der Gesellschaft Deutscher Nervenärzte.

Nach kurzen geschäftlichen Mitteilungen wird alsbald in die wissenschaftliche Tagesordnung eingetreten.

\section{Referat.}

a) Herr Emil Redlich-Wien: Die klinische Stellung der sogenannten genuineu Epilepsie.

Über die Stellung und den Umfang der sogenannten genuiuen Epilepsie herrscht selbst unter den Autoren, die sich für ihre strenge Festhaltung einsetzen, durchaus keine Übereinstimmung. Nach dem. Wortlaut können die synonymen Ausdrücke genuine, idiopathische oder essentielle Epilepsie ätiologisch, anatomisch oder pathogenetisch genommen werden. Man hat demnach, um zu einer Stellungnahme in dieser strittigen Frage zu gelangen, Ätiologie, Klinik und pathologische Anatomie der chronischen Epilepsie herangezogen. In ersterer Beziehung trann man die genuine Epilepsie als jene definieren, die eine unbekannte oder unklare Ätiologie hat, jedoch stellt ein solcher Standpunkt nach jeder Richtung hin nur ein Provisorium dar. Oder als jene, die aus der Anlage des Individuums hervorgeht. Dazu bedarf es freilich noch einer Unterscheidung zwischen disponierenden und auslösenden Ursachen der Epilepsie, wobei man vielfach aus prinzipiellen Grïnden eine Disposition auch dort, wo sie nicht nachweislich ist, voraussetzt. Was die Heredität betrifft, die man in der Ätiologie der genuinen Epilepsie an erste Stelle setzt, so halten die hohen. Zahlen, die man für eine hereditäre Belastung aufstellte, einer strengen Kritik nicht stand. Wichtig ist vor allem schwere Belastung und gleichartige und direkte Heredität. Die Epilepsie gehört aber nicht zu den direkten Erbkrankheiten, es wird höchstens eine Disposition für das Auftreten von epileptischen Anfällen vererbt, wobei andere Schädlichkeiten zur wirklichen Auslösung der Krankheit notwendig sind. Eine reine Keimschädigung durch Syphilis als Ursache der Epilepsie ist möglich, lässt sich aber bei dem heutigen Standpunkt unserer Syphiliskenntnisse nicht mit Sicherheit behaupten. Hingegen dürfte Keimschädigung durch Alkoholismus der Ascendenten, wenn auch gewiss nur in einer Eleinen Zahl von Fällen, anzuschuldigen sein.

Dieselben exogenen Schädlichkeiten können. beim Epileptiker Anfälle auslïsen, bei disponierten Individuen auch die Krankheit selbst, aber auch ohne Disposition die Krankheit direkt bedingen, wobei Zeichen einer grobanatomischen Hirnschädigung fehlen oder auch vorhanden sein können. Bei den akuten Infektionskrankheiten kommt es in dieser Hinsicht in erster Linie auf die Entwicklung 